\title{
The determination of cholesterol in serum by persulphuric acid oxidation
}

\author{
R. N. BEALE AND D. CROFT \\ From the Institute of Clinical Pathology and Medical Research, Department \\ of Public Health, Sydney, New South Wales
}

SYNOPSIS A method for the determination of total cholesterol in serum is described. Optimum conditions are found for the isolation of the steroid free from extraneous chromogens, and a new sensitive colour reagent is introduced. The procedure is convenient for routine application, the final colour being light-stable and reasonably time-stable at room temperature. Comparisons are made with one other method and with another colour reagent. It is concluded that, for accurate results, treatment of the serum with alcoholic alkali followed by extraction with a hydrophobic solvent is essential. The choice of the colour-developing reagent is then no longer dependent on specificity for cholesterol, but becomes inter alia a matter of ease of operation, and of sensitivity, stability, and reproducibility of the final colour. These points are discussed in relation to other methods.

The problem of determining accurately the total cholesterol content of blood serum may be resolved into two main parts, namely, the quantitative isolation of the cholesterol in a pure state and measurement of the isolated steroid by means of convenient developing reagents which lead to a sufficiently sensitive and stable colour.

The first part of the problem has been attacked in many ways by numerous workers, for example, by extraction with one or more organic solvents with concomitant precipitation of proteins (e.g., Bloor, 1916; Sackett, 1925); by similar extraction followed by precipitation of the cholesterol in the hydrolysed or unhydrolysed extract with digitonin (Sperry and Webb, 1950); by treatment with ethanolic potassium hydroxide followed by extraction (Abell, Levy, Brodie, and Kendall, 1952; Trinder, 1952; Anderson and Keys, 1956; Brown, 1959); or by combinations of these. Others (e.g., Zlatkis, Zak, and Boyle, 1953) have avoided isolation and added colour reagents directly to the serum.

The second part of the problem has also received much attention and since 1953 a number of methods have been published which are improvements on earlier techniques of colour development employing the Liebermann-Burchard reaction, the shortcomings of which are well documented.

The present work has led to a colorimetric method which incorporates the best features of those pre-

Received for publication 5 October 1961. viously described, reduces operations to a minimum, and introduces the use of persulphuric acid oxidation in an acetic-sulphuric acid medium. The colour produced is completely stable to light and sufficiently so to time, and the method has good sensitivity relative to others.

\section{REAGENTS}

Chemicals of A.R. quality should be used where possible.

1 ALCOHOL Absolute or $95 \%$ ethanol is suitable.

26 M POTASSIUM HYDROXIDE IN METHANOL Dissolve $84 \mathrm{~g}$. potassium hydroxide pellets in about $150 \mathrm{ml}$. methanol, cool and dilute to $200 \mathrm{ml}$. This reagent need not be filtered. It keeps for long periods in the refrigerator.

3 M POTASSIUM HYDROXIDE, ALCOHOLIC Dilute reagent (2) 1 in 5 with reagent (1). This is best done in small quantities as it deteriorates if ordinary ethanol is employed. The reagent need not be filtered.

M POTASSIUM HYDROXIDE IN PURIFIED ALCOHOL This is an alternative to the preceding reagent. Ninety-five to $100 \%$ ethanol is purified as follows. Add about $0.5 \mathrm{~g}$. 2:4-dinitrophenylhydrazine to each litre of alcohol followed by a few drops of concentrated sulphuric acid. The mixture is then refluxed for four to five hours (preferably with a gentle stream of nitrogen bubbling through the liquid), and left sealed from air overnight. The following day, the liquid is distilled with an efficient antisplash head or a fractionating column to avoid carryover of the hydrazine derivative. A small first fraction is 
rejected and about $80 \%$ of the remainder collected; nitrogen is again bubbled during distillation. The product is aldehyde-free. Dissolve $35 \mathrm{~g}$. potassium hydroxide pellets in aldehyde-free alcohol, cool, and dilute to $500 \mathrm{ml}$. with the alcohol. This reagent is fairly stable and will keep at least two months in the cold. Reject it when signs of yellowing appear.

4 Petroleum SPIRIt, Boiling-RANGe 60 to $80^{\circ} \mathrm{C}$.

5 ACETIC ACID Use glacial acetic acid; no purification is necessary.

6 PERSUlPhuric ACID REAGENT Dissolve $20 \mathrm{mg}$. potassium persulphate in $1 \mathrm{ml}$. cold water and add rapidly $100 \mathrm{ml}$. chilled concentrated sulphuric acid. Mix thoroughly. This reagent will keep for about three weeks in the refrigerator where it should be stored when not in use, and is rejected when the colorimeter reading for a $600 \mathrm{mg}$. \% cholesterol standard is markedly lower than with fresh reagent.

7 STANDARD CHOLESTEROL Dissolve $10 \mathrm{mg}$. pure cholesterol (e.g., Townson and Mercer's, chemically pure) in ice-cold petroleum spirit, and dilute to $100 \mathrm{ml}$. with cold solvent. Store in the refrigerator when not in use. Check the purity of the cholesterol by comparison with an authentic specimen, as some samples contain extraneous substances which are difficult to remove by recrystallization. Purity is checked by developing the colour with each sample and comparing the visible spectra, or the readings at about $498 \mathrm{~m} \mu$ if a filter instrument is used.

Aliquots of the standard solution are pipetted into test-tubes and the solvent evaporated in a water-bath at 80 to $100^{\circ} \mathrm{C}$. by blowing a gentle stream of air into the tube which is kept agitated. Nitrogen, instead of air, does not appear to be necessary. The dry standards are stable indefinitely, so that if desired a stock of these may be prepared.

\section{METHOD}

Measure $0.15 \mathrm{ml}$. serum into a glass-stoppered tube of about $20 \mathrm{ml}$. capacity. Then add $3 \mathrm{ml}$. M potassium hydroxide, mix and heat in a $60^{\circ} \mathrm{C}$. water-bath for 15 minutes. Cool the tube to room temperature and add $3 \mathrm{ml}$. water and $6 \mathrm{ml}$. light petroleum. Shake vigorously for 30 seconds to one minute and stand the tube for a moment. A clean separation occurs almost instantaneously. After the phases have separated, withdraw $2 \mathrm{ml}$. of the petrol layer and pipette into an ordinary rimmed test-tube. Evaporate the petrol as described previously. The residue is stable and may be stored indefinitely at this stage. For colour development add $5 \mathrm{ml}$. glacial acetic acid and allow the tube to stand for about five minutes with occasional shaking to dissolve the cholesterol residue. Then run $2.3 \mathrm{ml}$. persulphuric acid reagent down the wall of the sloped tube so as to form a layer beneath the acetic acid solution; allow a minute for drainage. Then shake the tube vigorously for about half a minute and transfer it without delay to a $37^{\circ} \mathrm{C}$. waterbath where it is allowed to incubate for five to 10 minutes.
Cool the tube to room temperature in water avoiding vigorous agitation and transfer the product to a colori- $\overline{0}$ meter tube or spectrophotometer cell. Read against a

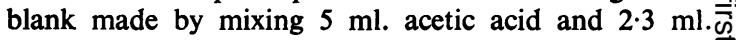
persulphuric acid reagent. The product has maximumabsorbance at $498 \mathrm{~m} \mu$; if a colorimeter is to be used afilter similar to the Ilford 623 is suitable.

For calibration measure 1,2 , and $3 \mathrm{ml}$. cholesterol $\frac{\omega}{\Phi}$ standard into rimmed test-tubes, evaporate the solvent and then proceed as for the colour development of the serum residue. These standards are equivalent to sera containing 200,400 , and $600 \mathrm{mg} . \%$ cholesterol. A cali-. bration graph may be used, but twice weekly checks with $\overrightarrow{\vec{\omega}}$ a duplicate standard are advisable. If preferred, water may be used as a blank in setting up the calibration and중 for future analyses.

\section{EXPERIMENTAL}

PREPARATION OF THE SERUM FOR COLOUR DEVELOPMENT In view of the variety of methods used for음 liberating cholesterol and its esters from serumconstituents, we attempted initially an assessment $\vec{z}$ of the various procedures for isolation such as those mentioned previously. Our objective was to discover $\vec{\theta}$ one which would separate the cholesterol either as such or mixed with its serum esters, would give $a_{\square}$ quantitative recovery of the total cholesterol, andO would lead to an isolated product free of extraneous chromogens; in addition it was desirable that anyo coloured substances such as bilirubin or haemo-市 globin should be left behind during the isolation procedure. The criterion used was that based on ano음 examination of the visible absorption spectra of the colours derived from treated serum and from pure

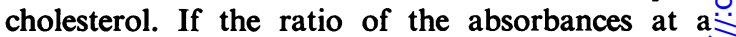
series of corresponding wavelengths remained con-응 stant, then it could be concluded either that the $\delta$ cholesterol had been obtained in a pure state (or 3 . together with esters which gave the same absorption spectra on a molar basis as free cholesterol) or that the method of colour development was specifico for cholesterol, or both. Purity of the isolated cholesterol was gauged by colour development of dried extracts and standards with a modified $\bar{N}$ Liebermann-Burchard reagent (Anderson and Keys), with the reagents of Zlatkis et al. as modified by 0 Henly (1957) but without purification of the acetic N acid, and also with our reagents.

The methods of extraction which were investigated involved addition of acetic acid and centrifugation $; \frac{\bar{C}}{\bar{C}}$ addition of acetic and trichloracetic acids followed by centrifugation; hot and cold extraction with $\square$ various solvents or mixtures of solvents; and lastly $\bar{O}$ treatment with aqueous, methanolic or ethanolic potassium hydroxide, or with mixtures of these, at $\overrightarrow{\mathbb{D}}$ various temperatures and for varying times, followed $\frac{\varrho}{\sigma}$ by petrol extraction. 
Examination of the spectra of the final products from the first three types of extraction procedure showed lack of correspondence with the spectrum from pure cholesterol; in all cases extraneous absorption was observed giving falsely high values, so that such methods were rejected as unsatisfactory for accurate work. Since the result was much the same for any of the three sets of colour reagents used it appears that none of these methods of colour development is specific for cholesterol; contribution of non-reacting colour from the serum itself is a further possible source of positive error. These findings are supported by the work of Morris (1959) who carried out a painstaking comparison of six methods. If the work of Schoenheimer and Sperry (1934) and of Sperry and Webb (1950) is taken as a base-line for comparative purposes it is apparent from Morris's data that the methods of Pearson, Stern, and McGavack (1953), Zlatkis et al. (with or without purified acetic acid), and Sackett give mean values significantly higher; furthermore, acid extractants lead to higher values than neutral organic extractants.

Attention was therefore directed to treatment of the serum with alkali and petrol, and by this means satisfactory results could be achieved under optimum conditions (the optima not being particularly critical, as will be described). It was found possible to produce spectra from the extracts which were virtually identical with those from pure cholesterol (Fig. 1). Since the colour reagents mentioned above are not specific, it must be concluded from this that by treatment with alcoholic potassium hydroxide and extraction with petrol the cholesterol is isolated from serum in almost pure form, the impurities having negligible chromogenic potentialities with at least some colour reagents. This finding confirms

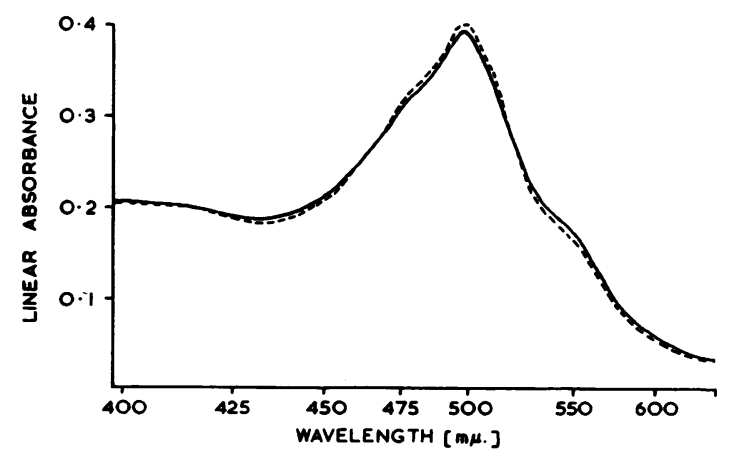

FIG. 1. Absorption spectrum of colour from a $270 \mathrm{mg} . \%$ cholesterol standard (full curve) and the same from $a$ processed serum, showing freedom from extraneous absorption (broken curve). the work of Abell et al. who showed by countercurrent distribution that the cholesterol obtained by their procedure is $99 \%$ pure.

A number of variations in detail for alcoholic alkali treatment have been published. Trinder heats to boiling with $0.5 \mathrm{~N}$ potassium hydroxide and discontinues as soon as steady boiling occurs, while others (e.g., Anderson and Keys; Sobel, Goodman, and Blau, 1951; and Brown) heat at $37^{\circ}, 45^{\circ}$, or $65^{\circ} \mathrm{C}$. for 90 minutes or less. Little specific investigation of the variables such as alkali concentration, temperature and time of heating, and nature of solvent seems to have been published, although according to Bloor, Pelkan, and Allen (1922) heating with strong alkali is dangerous, a contention which seems to have passed unchallenged subsequently. Some of the parameters were explored, therefore, in order to determine the optimum conditions for isolation. The colour reagent described under 'Method' was used.

Time of heating at $60^{\circ} \mathrm{C}$. Treatment with $\mathrm{M}$ potassium hydroxide in alcohol ( $95 \%$ or more) gave $55 \%$ maximum absorption at $498 \mathrm{~m} \mu$ for an immediate extraction, $94 \%$ after two minutes, and maximum colour for between eight and 16 minutes' extraction; very slightly lower values were obtained at 24 and 30 minutes. The best spectral purity relative to a standard was obtained after 15 to 20 minutes' heating and this period is recommended.

Concentration of alkali Aliquots of a serum were incubated at $60^{\circ} \mathrm{C}$. for 15 minutes with $0 \cdot 5,1,2$, and $4 \mathrm{M}$ alcoholic potash and processed as described. No significant difference between any of the resultant spectra was observed. $M$ potassium hydroxide was chosen to allow for any possible weakening of the solution with time, due to carbonate formation. At $60^{\circ} \mathrm{C}$., therefore, considerable stability of cholesterol in the presence of alkali is indicated.

Heating temperature Treatment with $0.5 \mathrm{M}$ alcoholic alkali at the boiling point was the only other temperature variant investigated. Under Trinder's conditions, described previously, a somewhat lower absorption at $498 \mathrm{~m} \mu$ was obtained compared with the present method. If, however, the mixture was refluxed for two to eight minutes, a result not significantly different from 15 minutes' treatment at $60^{\circ} \mathrm{C}$. was obtained. A comparison with Trinder's method is described later.

Variation of solvent When aqueous or methanolic potash of any concentration was used, poor recoveries of serum cholesterol were obtained. On the other hand addition to ethanolic potassium hydroxide of water up to $16 \% \mathrm{v} / \mathrm{v}$ or of methanol up to $17 \% \mathrm{v} / \mathrm{v}$ had no effect on the final result.

THE COLOUR DEVELOPING REAGENT Many colour reactions for cholesterol have been described, 
mostly employing a combination of two or more of the solvents acetic acid, acetic anhydride, sulphuric acid, chloroform, and more recently, ethylene dichloride and acetyl chloride. Reaction is carried out in the presence of an oxidizing agent or agents such as sulphuric acid, ferric chloride, or perchloric acid. The most sensitive so far described for analytical purposes is that of Brown who modified Trinder's procedure by replacing sulphuric acid with perchloric acid. Both these reactions use ethylene dichloride which is toxic, and acetyl chloride, the vapour of which is volatile and hydrolyses readily in air to acetic and hydrochloric acids. The purity of ethylene dichloride appears to be critical for colour sensitivity and stability in Brown's method. When we used laboratory reagent grade material which had no processing other than redistillation,with rejection of the first and last runnings, the colour faded obviously, a drop of $25 \%$ being observed in two hours when the solution stood in diffuse daylight. In direct sunlight the colour faded to yellow in 25 minutes. When the solvent was purified and dried as recommended and clean, dry apparatus used, with precautions to exclude moisture, considerable improvements in stability were obtained for both sera and standards. After 30 minutes in the dark only about $2 \%$ of the colour faded; after an equivalent time in diffuse daylight, a drop in colour of about $4 \%$ was found; the blank was unchanged. The colour was decidedly unstable to sunlight, 10 minutes' irradiation causing a decrease of 6 to $10 \%$ in the colorimeter reading.

Ferric chloride oxidation in acetic-sulphuric acid (Zlatkis et al., 1953) is an amenable reaction for routine purposes if used in conjunction with the serum treatment described under 'Method'. It has the advantage of employing a stable oxidizing colour reagent (if modified by incorporating the ferric chloride in the acetic acid rather than the sulphuric acid) but the disadvantage of producing a colour which is markedly unstable to light. After 15 minutes in bright diffuse daylight at room temperature the solution gave a spectrum which showed a drop of $9 \%$ in maximum absorbance at $560 \mathrm{~m} \mu$ when compared with the dark-developed spectrum. After 11 minutes in direct bright daylight the original spectrum changed markedly and a brown colour was formed. If the colour is developed in the light, it has quite a different shade to a duplicate developed in darkness. On the other hand, the time stability of the colour in darkness is good, little change being observed over several hours. Another disadvantage, as pointed out by Morris, is the tendency for stationary air bubbles to form in the viscous acid mixture. These are difficult to remove except by centrifugation and interfere during colorimetry, giving erroneously high readings. At lower sulphuric acid concentrations less viscous media are produced but there is considerable loss of sensitivity.

In the present work we investigated a number of combinations of solvents such as those referred to earlier, these being used in the presence or absence of an oxidant. Of the solvents, mixtures of acetic and sulphuric acids were considered to be the most convenient for several reasons; they are relatively inexpensive and readily available in large quantities in most laboratories; they may be used under some conditions, such as the present ones, without timeconsuming purification; they are relatively nonvolatile and they give a medium with good potentiality for sensitive colour reactions. In conjunction with such a solvent mixture a wide range of oxidants was examined and those were selected which produced the most intense colour. Three gave acceptable results, namely, ferric ion, periodate, and persulphate. Ferric salts were excluded by reason of the light instability and viscosity of the coloured product; periodate was somewhat inferior to persulphate since over-oxidation occurred and colour faded. Persulphate was therefore chosen since it was free from these disadvantages.

Variation in concentration of constituents Having determined the optimum proportions of acetic acid, sulphuric acid, and persulphate, we varied each in turn from the optimum. A deficiency of sulphuric acid led to considerable loss of sensitivity and an excess gave too viscous a reagent and too high an initial oxidizing temperature. The optimum desirable in order to avoid both these factors was found to be 5 volumes of acetic acid to 2 to $2 \cdot 4$ volumes of sulphuric acid. These proportions gave maximum colour sensitivity at $498 \mathrm{~m} \mu$ and a medium which, under the conditions used, did not often occlude air bubbles unless handled too vigorously after cooling to room temperature. The concentration of persulphate was not critical; decreasing concentra- $\frac{0}{3}$ tion led to decrease in sensitivity and a different $D$ absorption spectrum, while excess persulphate led to over-oxidation and loss of colour. The optimum $N$ range for a fresh reagent was found to be 10 to $25 \mathrm{mg}$. per $100 \mathrm{ml}$. sulphuric acid.

Conditions for colour development The marked $N_{\omega}$ rise in temperature on mixing the persulphuric and acetic acid layers assists in the rapid development of 0 an orange-red colour in the presence of cholesterol. $\overparen{\Phi}$ The process is then completed by maintaining the $\stackrel{\infty}{+}$ mixture above room temperature for a time. At $60^{\circ} \mathrm{C}$. no more than two to three minutes is required but $\stackrel{\vec{\Phi}}{\circ}$ this temperature causes small changes in the spectrum $\cong$ of the coloured product. Incubation at $37^{\circ} \mathrm{C}$. for five $\stackrel{\mathbb{Q}}{\mathbb{Q}}$ to 10 minutes is as effective in producing maximum colour and during this time no significant change in 
the spectrum is observed, the absorption at $498 \mathrm{~m} \mu$ being constant. The lower temperature is used, therefore, and the solutions are then ready for colorimetry after cooling to room temperature.

Stability of colour The colour formed is insensitive to light: after 10 minutes' irradiation in bright sunlight outside the laboratory no significant change in the spectrum was observed. The same result was found after 90 minutes' irradiation in a combination of direct sunlight and bright daylight. After a further 17 hours in diffuse daylight and darkness at about $20^{\circ} \mathrm{C}$., the spectrum showed some change, there being a drop in absorbance at $498 \mathrm{~m} \mu$ of 4 to $5 \%$ and an increase of the same order in the region of $430 \mathrm{~m} \mu$. The time of reading is not critical, therefore, but for the most accurate results measurements are best made within a few hours of colour development.

Obedience to Beer's law Cholesterol standards up to a serum equivalent of $600 \mathrm{mg} . \%$ (up to $0.3 \mathrm{mg}$.) were developed and the absorbances at $498 \mathrm{~m} \mu$ (or their colorimeter equivalent) were measured on various instruments. The readings on a moderately high resolution recording spectrophotometer showed obedience to Beer's law up to at least the highest level measured. The calibration graph is non-linear at the higher level for most colorimeters for the usual reason that light scatter in their optical systems lowers readings at the top end of the scale. It is probably advisable therefore to confine colorimeter readings to no more than $70 \%$ full-scale deflection. Within this range linearity is maintained for an average instrument.

\section{RESULTS}

RECOVERY OF CHOLESTEROL Since no way could be envisaged of incorporating cholesterol into serum which would represent an approach to the true state of distribution, it was not considered meaningful to add cholesterol to serum in order to carry out recovery experiments in the usual fashion. Instead, several dried standards were processed as for serum in order to discover whether losses occurred during alkali treatment and solvent extraction or through chemical breakdown of cholesterol. The mean recovery of pure steroid was quantitative $(100 \pm 2 \%)$. This is not to say that those from sera would be as satisfactory, but other studies would be required to decide this point. In the present work we used the method of alkali treatment which appeared to give the maximum and most consistent recovery of serum cholesterol and compared this with the method of Trinder and with a modification of that of Zlatkis et al. In addition, the colour reagents developed by Trinder and by us were employed in combination with both methods of alkali treatment. The results for a number of sera determined in these latter ways are shown in Table I; all analyses were carried out in duplicate and the individual means taken for comparative purposes.

\section{TABLE I}

COMPARISON OF SERUM CHOLESTEROL LEVELS OBTAINED USING COMBINATIONS OF TWO ALKALI TREATMENTS AND TWO COLOUR REAGENTS

\begin{tabular}{|c|c|c|c|c|}
\hline \multirow{2}{*}{$\begin{array}{l}\text { Serum } \\
\text { No. }\end{array}$} & \multicolumn{2}{|c|}{ Trinder Colour Reagent } & \multicolumn{2}{|c|}{ Present Colour Reagent } \\
\hline & $\begin{array}{l}\text { Trinder } \\
\text { Isolation } \\
(m g . \%)\end{array}$ & $\begin{array}{l}\text { Present } \\
\text { Isolation } \\
(m g . \%)\end{array}$ & $\begin{array}{l}\text { Trinder } \\
\text { Isolation } \\
\text { (mg. \%) }\end{array}$ & $\begin{array}{l}\text { Present } \\
\text { Isolation } \\
(\mathrm{mg} . \%)\end{array}$ \\
\hline 263 & 324 & 350 & 319 & 350 \\
\hline 228 & 141 & 138 & 134 & 137 \\
\hline 155 & 116 & 121 & 129 & 131 \\
\hline 309 & 216 & 223 & 218 & 226 \\
\hline 230 & 204 & - & - & 276 \\
\hline 326 & 203 & - & - & 264 \\
\hline 328 & - & 280 & - & 271 \\
\hline 339 & - & 338 & - & 342 \\
\hline 359 & 141 & - & - & 151 \\
\hline 354 & 83 & - & - & 97 \\
\hline
\end{tabular}

A number of points of interest emerge from Table I when the mean values for corresponding groups of sera are compared. Either colour reagent gives, for the same alkali treatment, means in close accord. When the two procedures for isolating the cholesterol are compared, however (using either colour reagent), the mean serum level with Trinder's treatment is about $90 \%$ of that using the present one.

In Table II are shown the comparative results for sera analysed by the present procedure and by a modification of Henly's development of the Zlatkis method. No purification of the acetic acid A.R. was carried out and the cholesterol was isolated as described in this paper. The ferric chloride was incorporated in the acetic acid as recommended by Henly. Procedures somewhat similar to the ZlatkisHenly modification have been described recently by Mann (1961) and by Connerty, Briggs, and Eaton (1961), all of whom, like Zlatkis et al., use ferric ionsulphuric acid-acetic acid reagents. For each sample referred to in Table II, four determinations were carried out using a fresh aliquot of serum for each of the four analyses. In the modified Zlatkis-Henly method, any bubbles in the coloured product were removed by light centrifugation of the colorimeter tubes. Colour development was carried out in a dark cupboard and subsequent operations were performed as soon as possible in subdued light. As is apparent from Table II, no significant difference between the two sets of values was found. 
TABLE II

COMPARISON OF PRESENT METHOD WITH A COMBINED MODIFICATION OF THAT OF ZLATKIS et al. (1953) AND OF HENLY (1957)

\begin{tabular}{|c|c|c|c|c|c|}
\hline \multirow{2}{*}{$\begin{array}{c}\text { Serum No. } \\
818\end{array}$} & \multicolumn{2}{|c|}{$\begin{array}{l}\text { Present } \\
(m g . \%)\end{array}$} & \multicolumn{2}{|c|}{$\begin{array}{l}\text { Zlatkis-Henly } \\
\text { (mg. \%) }\end{array}$} & \multirow{2}{*}{$\begin{array}{c}\begin{array}{c}\text { Percentage } \\
\text { Difference }\end{array} \\
0\end{array}$} \\
\hline & $\begin{array}{l}166 \\
166\end{array}$ & 166 & $\begin{array}{l}167 \\
165\end{array}$ & 166 & \\
\hline 952 & $\begin{array}{l}162 \\
159\end{array}$ & 161 & $\begin{array}{l}159 \\
169\end{array}$ & 164 & +1.9 \\
\hline 965 & $\begin{array}{l}198 \\
196\end{array}$ & 197 & $\begin{array}{l}176 \\
184\end{array}$ & 180 & -8.6 \\
\hline 988 & $\begin{array}{l}206 \\
207\end{array}$ & 207 & $\begin{array}{l}203 \\
219\end{array}$ & 211 & +1.9 \\
\hline 024 & $\begin{array}{l}181 \\
174\end{array}$ & 178 & $\begin{array}{l}173 \\
179\end{array}$ & 176 & $-1 \cdot 1$ \\
\hline 950 & $\begin{array}{l}150 \\
154\end{array}$ & 152 & $\begin{array}{l}152 \\
147\end{array}$ & 150 & $-1 \cdot 3$ \\
\hline 563 & $\begin{array}{l}209 \\
213\end{array}$ & 211 & $\begin{array}{l}208 \\
197\end{array}$ & 203 & -3.8 \\
\hline 025 & $\begin{array}{l}321 \\
314\end{array}$ & 318 & $\begin{array}{l}323 \\
316\end{array}$ & 320 & +0.6 \\
\hline 576 & $\begin{array}{l}104 \\
104\end{array}$ & 104 & $\begin{array}{l}109 \\
110\end{array}$ & 110 & +5.8 \\
\hline \multirow[t]{2}{*}{599} & $\begin{array}{l}216 \\
208\end{array}$ & 212 & $\begin{array}{l}213 \\
218\end{array}$ & 216 & +1.9 \\
\hline & & & \multicolumn{2}{|c|}{ Mean difference } & $-0.3 \%$ \\
\hline
\end{tabular}

REPRODUCIBILITY This was assessed for the present method by processing 12 pairs of standards and 13 pairs of test sera and applying the statistic

$$
\text { (S.E.M. }^{2}=\frac{\Sigma \Delta^{2}}{2 \mathrm{~N}}
$$

where S.E.M. = standard error of measurement

$$
\begin{aligned}
\Delta= & \text { percentage difference between one } \\
& \text { value of the pair and its mean } \\
\mathrm{N} & =\text { number of pairs }
\end{aligned}
$$

The S.E.M. was $\pm 1.0 \%$, so that the extreme confidence limits for a single test are about $\pm 3.0 \%$; that is to say, in $99.7 \%$ of cases the measured value should be no more than $\pm 3 \%$ away from the 'true' value. Morris found Trinder's method to be the most reproducible of those he investigated, the S.E.M. being $\pm 1 \cdot 2 \%$; our value for Trinder's method was in close agreement with this, being $\pm 1 \cdot 4 \%$. The reproducibility of the present method therefore corresponds closely with his, From Table II it appears that ferric chloride oxidation gives results not quite so reproducible as those above, the S.E.M. being $\pm 1.5 \%$. Using Zlatkis' original method, Morris found a S.E.M. of $\pm 1.6 \%$ for unpurified acetic acid. As described under 'Experimental', the method of isolation is such that, with the present colour reagents, no interfering chromogens remain; neither is there the problem of possible variations in chromogenic power of different esters if complete hydrolysis is assumed. With deliberately haemolysed or highly icteric sera no interferences due to haem compounds or bilirubin were observed.
CHOLESTEROL ESTERS The spectra of the coloured derivatives from some esters of cholesterol were measured before and after alkali treatment under the described conditions. In all cases the spectra had the same shape with a maximum at $498 \mathrm{~m} \mu$. However, the band intensities (or the absorbance at $498 \mathrm{~m} \mu$ ) varied slightly from that found for cholesterol. Small differences were observed for oleate $(97 \pm 2 \%$ of cholesterol), acetate (104 $\pm 2 \%$ of cholesterol), and for stearate (98 $\pm 2.0 \%$ of cholesterol), the comparisons being made on a molar basis. It would appear that, with the method described, the presence of even considerable amounts of esters in the final extracts would have little effect on the values found for serum levels.

SENSITIVITY The most sensitive method so far reported is that of Brown, which he states to have 8.2 times the sensitivity of the Liebermann-Burchard procedure described by Abell et al. On the same basis Trinder's method gives a factor of 4.6 and that of Zlatkis et al. is 7.3. The present method is intermediate between those of Zlatkis et al. and Trinder in this respect, having a factor of 6.2 when equal concentrations of cholesterol in the final product are compared. In practice, all these more sensitive procedures give good colorimeter readings for quite small amounts of serum. For a $200 \mathrm{mg}$. \% cholesterol standard $(100 \mu \mathrm{g}$. cholesterol) our end product has a linear absorbance of about 0.31 at $498 \mathrm{~m} \mu$ for a $1 \mathrm{~cm}$. light path with a reagent blank in the comparison cell. Thus, as little as $30 \mu \mathrm{g}$. cholesterol can readily be measured.

\section{DISCUSSION}

It emerges that the main requisite in determining cholesterol is its quantitative liberation from the serum in a sufficiently pure condition, so that, with any chromofacient, the colour is due to cholesterol 오 alone. This becomes necessary since, as may be $D$ deduced, for example, from Morris's data and as appears from the present work, most colour reagents $N$ are non-specific for cholesterol. Thus direct methods fail to meet the situation; procedures involving only $N$ acid or solvent treatment of serum are inadequate $\omega$ also, since extracted chromogenic substances, sometimes in considerable quantity, again contaminate 0 the cholesterol. When the Liebermann-Burchard $\bar{\Phi}$ reaction was applied to solvent-extracted and also $\stackrel{\mathscr{?}}{+}$ to alkali-treated serum, only a small but significant $\underline{T}$ difference was found between the spectra of the resulting colours, so that the Liebermann-Burchard $\stackrel{\cap}{\Omega}$

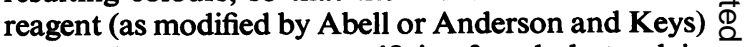
approaches nearest to specificity for cholesterol in $\bar{\sigma}$ the presence of solvent extractable contaminants. 
However, even if acceptable in this respect, the wellknown limitations of the reaction in other respects suggest the need for improved methods employing more sensitive and convenient reagents and producing more stable resultants.

Since it is difficult to ascertain whether or not recoveries from sera are quantitative, the present approach has been to obtain maximum sample colour together with correspondence of spectra from sera and pure cholesterol. Such criteria could be satisfied by treatment under the conditions corresponding or approximating to those described under 'Method'. When this treatment is combined with extraction by a hydrophobic solvent virtual freedom from extraneous chromogens is achievable with the reagents described under 'Method'.

In our hands, when Trinder's method of treatment was closely followed, apparently low recoveries of cholesterol resulted; although if the alkali treatment was continued at the boiling point for as little as two minutes, close correspondence of serum levels was obtained by both methods.

Little appears to be known concerning the nature of cholesterol binding in serum, but the purpose of alkali treatment is sometimes stated to be that of ester hydrolysis. It would appear, however, as pointed out by Abell, that a more important function of such procedures is to liberate the cholesterol and its esters from their complexes and combinations in serum, saponification being a concomitant process; otherwise it is difficult to account for the findings in Table I, as it has been shown that with our reagents at least some esters have approximately the equivalent chromogenic power of cholesterol. A minimum time appears necessary to complete this process of liberation; thus, for example, Anderson and Keys use $2 \%$ alcoholic potash at $37^{\circ} \mathrm{C}$. for 90 minutes. Brown employs $2.5 \%$ alkali at $65^{\circ} \mathrm{C}$. for 30 to 60 minutes, while in the present method we find $M$ alkali at $60^{\circ} \mathrm{C}$. for 15 minutes to be adequate; the latter conditions would be approximately equivalent to two minutes' refluxing at $90^{\circ} \mathrm{C}$., if one assumes a doubling of reaction rate for each $10^{\circ}$ rise in temperature. This may explain our lower results with Trinder's method since less than two minutes' refluxing is allowed. Thus, provided that treatment time exceeds an irreducible minimum, the conditions of alkaline digestion are by no means critical and comparable values are then obtainable with any one of several colour reagents.

All of the latter so far discussed have been shown to be non-specific for cholesterol, but with extraction procedures such as that described, interfering chromogens are of negligible proportions. The choice of reagent can subsequently be resolved into questions of sensitivity, reproducibility, ease of handling and preparation, and time- and lightstability. In this latter respect the colour reagent described under 'Method' is the only one of those we have examined which gives a product which is quite stable to light and at the same time does not necessarily involve special purifications of solvents.

No comparison between the present method and a digitonin precipitation method has been attempted, but at first sight evidence could be adduced that such methods lead to low values for serum cholesterol levels. Thus Anderson and Keys find that their K5 (alkali treatment) method gives values some $3 \%$ higher than the digitonin procedure of Foldes and Wilson (1950). Since, as appears from the present work, this cannot be attributed to non-specificity of the K5 colour reagent, their alternative explanation of losses due to slight solubility of cholesterol digitonide would seem possible. On the other hand, Morris obtains values $1 \%$ lower by Anderson and Keys' than by Sperry and Webb's method. However, his values for Trinder's method, which would be expected to agree with Anderson and Keys', are significantly higher than either the above-mentioned ones by 6 to $7 \%$. It would be expected, therefore, from this evidence that our values also would be higher than those obtained using a digitonin procedure by at least this amount. Assessment is made more difficult by the fact that when Trinder compares his method with that of Sperry and Webb, he obtains very close agreement for the mean of 10 sera. One possible explanation would be that Morris used slightly longer periods of alkali treatment than did Trinder, which, as we have shown, could lead to values up to $10 \%$ higher. Further critical comparisons of the various methods are indicated.

This work is published with the approval of the Director of State Health Services, Department of Public Health, New South Wales.

\section{REFERENCES}

Abell, L. L., Levy, B. B., Brodie, B. B., and Kendall, F. E. (1952). J. biol. Chem., 195, 357.

Anderson, J. T., and Keys, A. (1956). Clin. Chem, 2, 145.

Bloor, W. R. (1916). J. biol. Chem., 24, 227.

-, Pelkan, K. F., and Allen, D. M. (1922). Ibid., 52, 191.

Brown, W. D. (1959). Aust. J. exp. Biol., 37, 523.

Connerty, H. V., Briggs, A. R., and Eaton, E. H. (1961). Clin. Chem., $7,37$.

Foldes, F. F., and Wilson, B. C. (1950). Analyt. Chem., 22, 1210.

Henly, A. A. (1957). Analyst, 82, 286.

Mann, G. V. (1961). Clin. Chem., 7, No. 3, p. 275.

Morris, T. G. (1959). J. clin. Path., 12, 518.

Pearson, S., Stern, S., and McGavack, T. H. (1953). Analyt. Chem., $25,813$.

Sackett, G. E. (1925). J. biol. Chem., 64, 203.

Schoenheimer, R., and Sperry, W. M. (1934). Ibid., 106, 745.

Sobel, A. E., Goodman, J., and Blau, M. (1951). Analyt. Chem., 23, 516.

Sperry, W. M., and Webb, M. (1950). J. biol. Chem., 187, 97.

Trinder, P. (1952). Analyst, 77, 321.

Zlatkis, A., Zak, B., and Boyle, A. J. (1953). J. Lab. clin. Med., 41, 486. 